\title{
ZUR FARN-FLORA
}

DER

S U N D A-I N S E L N

VON

H. C H R I S T.

Fxtrait des Annales du Jardin Botanique de Buitenzorg.

Vol. XIII, pag. 90-96.

LEIDE. - E. J. B R ILL. 1895. 


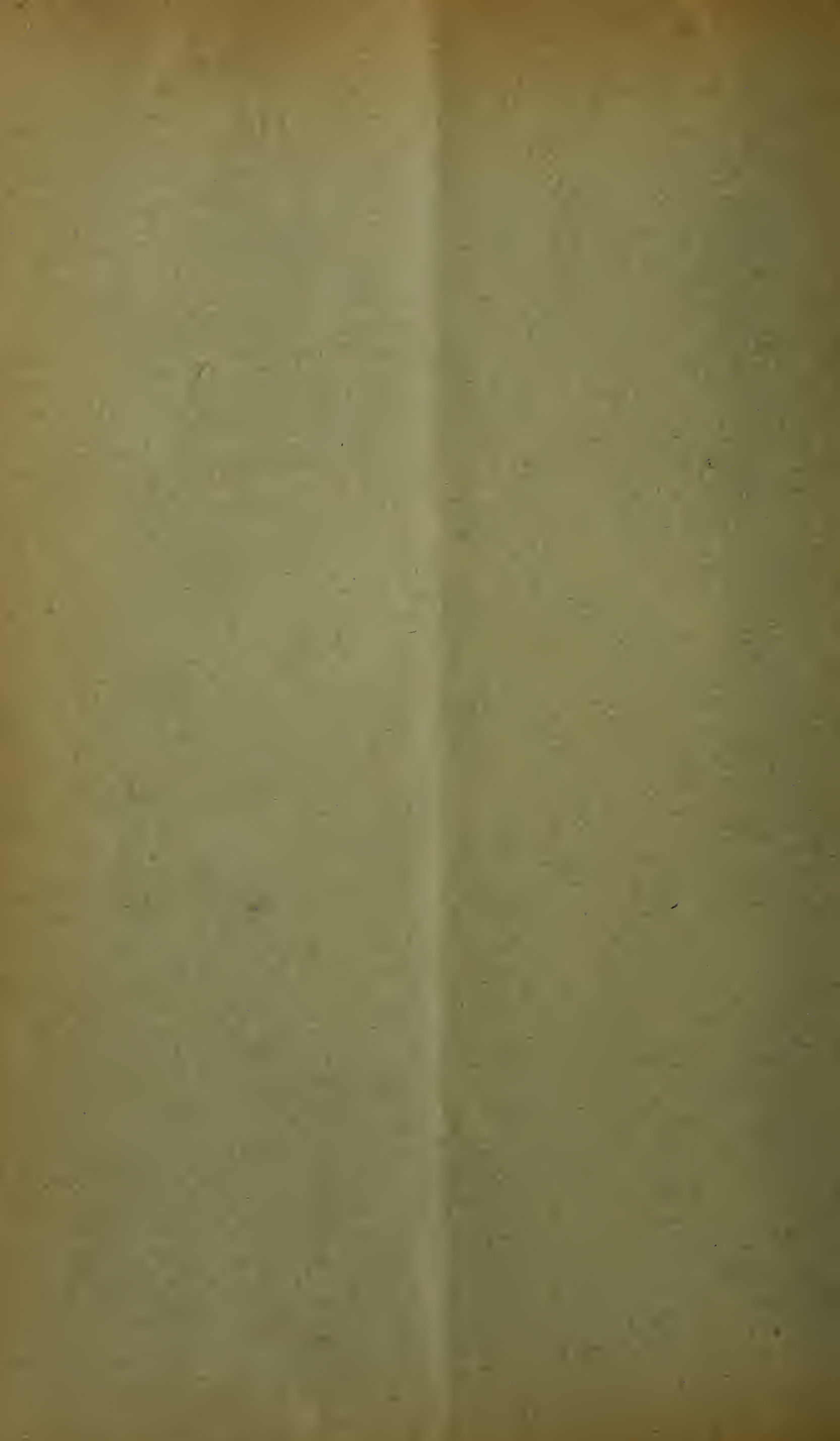





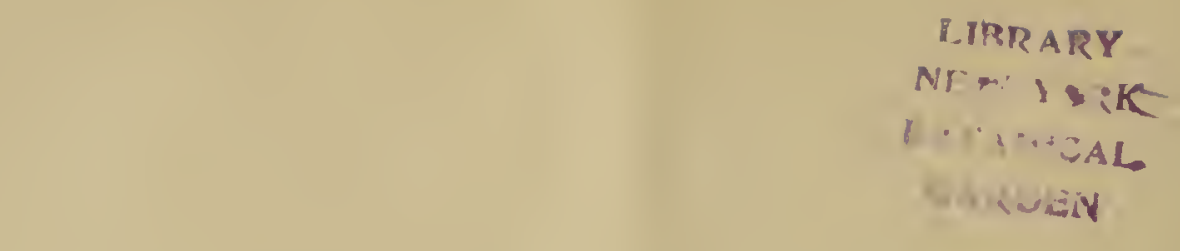

ZUR FARN-FLORA DER SUNDA-INSELN.

VON

Dr. H. CHRIST.

I. Diplazium speciosum Blume Enum. Plant. Javae fasc. II, pg. 193. Christ, Ueber einige Javanische Arten von Diplazium. Annales du Jardin bot. de Buitenzorg. XII. 21\%.

II. T'eratophyllum Metten = Acrostichum (Stenochlaena I. Sm.).

G. Karsten behandelt in seinen morpholog. u. biologischen Untersuchungen über einige Epiphytenformen der Molukken (in Annales du Jardin bot. de Buitenzorg XII. 143.) eine Pflanze von Amboina mit dimorphen Blättern, die er der von Mettenius aufgestellten Gattung Teratophyllum zurechnet. Diese Identification ist durchaus richtig: es fragt sich aber, ob dies Genus Teratophyllum aufrecht, zu erhalten ist, ob nicht vielmehr diese Pflanze zu einem längst bekannten Acrostichum zu-ziehen ist.

Für mich ist diese Frage durchaus zu bejahen:

es handelt sich hier lediglich um ein besonderes, vollkommenes Entwicklungsstadium der Acrostichum scandens (I. Sm.) und sorbifolium L. aus der Gruppe Stenochlaena I. Sm. (Lomariopsis Fée).

Bekanntlich haben die sonderbaren, in ihrer Textur an Trichomanes erinnernden Niederblätter dieser Kletterpflanzen mehrfach die Aufmerksamkeit auf sich gezogen. Schon Blume in dem $2^{\text {ten }}$ Heft der Enum. plant. Javae 182\%. S. 205 beschreibt kurz unter Lomariae Dubiae 2. Pflanzen, die er Lom. aculeata u. gracilis nennt, mit kletterndem und stachligem Caudex und mit Fiedern, die theils einfach und ganzrandig, theils zweifach fiederspaltig sind, und W. I. Hooker in spec. fil V. 243. 1864. 
vermuthet, dass das abnorme Formen des Acrostichum sorbifolium E. seien.

Der erste genauer erörterte Fall dieser Art war die von Bory de St. Vincent in Duperrey's Voyage de la Coquille Bot. 2\%3, Tab. 3\%. f. abgebildete, von ihm Scolopendrium Durvillei benannte Pflanze, welche Du Mont d'Urville auf Ualan gesammelt hatte. Nach den ebenda von Martens fil. auf der Kotzbue'schen Expedition gefundenen Exemplaren beschrieb sie Kunze in Schluhr's Suppl. 1840-184y Tab. V. S. 9. Diese Pflanze zeigt kletternden Caudex und gefiederte Wedel, deren Endsegmente ungetheilt, und in ihrem Bau identisch mit Acrostichum sorbifolium sind, während die Seitensegmente sich in doppelt fiederspaltige Gebilde vom Aussehen eines Trichomanes der Radicans-Gruppe auflösen. Wegen einiger unregelmässiger Sporangien-Gruppen, die längs den Seitennerven der Endsegmente verlaufen, brachten sowohl Bory als Kunze diese Pflanze zu Scolopendrium.

Ein fernerer Fall dieser Art ist das von W. I. Hooker in spec. fil. I. 1846. Tab. 61 D. Seite 196 geschilderte sterile Stück eines Farns, der denselben kriechenden Caudex darstellt, jedoch mit kurzen Stacheln versehen und dessen Wedel lediglich eine sehr zusammengesezte regelmässig doppelt bis dreifach fiederspaltige Trichomanesform zeigt, ohne das einfache Endsegment der D'Urville'schen und Martens'schen Pflanze. Diese Form ist von Penang l. Wallich und wurde von diesem, ohne Zweifel wegen der regelmässigen Fiedertheilung, in seinem Catalog N. 148 als Davallia achilleaefolia bezeichnet. Im Text citirt Hooker die Beobachtung von I. Smith (in Enum filic. Philipp. in Hook Journ. of Bot. III. 401.) zu Exemplaren der Stenochlaena scandens I. Sm. von Cuming aus den Philippinen, an denen ein gleiches Gebilde sich findet, das Smith als abnorme Wedel bezeichnet, die sich an verlängerten stachellosen oder auch bestachelten Theilen des Stipes entwickeln. Smith findet diese Gebilde analog den trichomanoiden Ansätzen am Stipes der Hemitelia capensis Br., und Hooker schliesst sich dieser Ansicht "heartily" an. Auch im V. Band der spec. fil. N. 242. hält er es für wahrscheinlich, dass die von Fée als besondere Arten von Lomariopsis (ludens, 
variabilis, Smithii, spinescens) beschriebenen Formen nichts sind als Pflanzen von Acrostichum sorbifolinm L. u. scandens (I. Sm.) welche neben normalen Wedeln fiederspaltige Niederblätter zeigen. Hooker zieht auch mit gleicher Wahrscheinlichkeit sein Polypodium binerve von Madagaskar (Sp. fil. IV. 1\%5. Tab. 273. B.) als eine einfach fiederspaltige Form zu A. sorbifolium L.

Für mich ist nun die Zugehörigkeit dieser, von Karsten vortrefflich nach Textur und biologischer Bedeutung beschriebenen Form mit trichomanoiden Niederblättern zu jenen beiden, so nahe verwandten Acrostichum eine ausgemachle Thatsache, und es ist das Mettenius'sche Genus Teratophyllum zu unterdrücken, wie dies schon in der Synops. fil. Ed. II (1873.) N. 412. ausgesprochen ist. Ich stütze mich dabei auf Material, das ich Herrn Karsten selbst verdanke, und das aufs genaueste mit sehr schönen Exemplaren von A. sorbifolium L. stimmt, welche Bischof Hose in Borneo: Sarawak 1894. sammelte und die er mit der Bemerkung begleitete: showing the curious tripinnatifid basal fronds which are common. Den Exemplaren liegt ein fruchttragender Wedel bei, welcher nicht den geringsten Zweifel übrig lässt. Auch zeigt die Abbildung Karsten's Taf. 14. 215. N. 26. $2 \%$ u. 28. aufs genaueste dies Acrostichum.

$\mathrm{Ob}$ nun solche Formen zu A. sorbifolium L. oder zu A. scandens (J. Sm.) gehören, ist nicht immer leicht zu entscheiden. Am besten leitet noch der feine Nerv, der bei scandens längs dem Mittelnerv der Fieder verlüuft, von dem aus erst die senkrecht abgehenden Seitennervillen entspringen, und dann sehr deutlich ist, wenn er sich vom Mittelnerv ein wenig entfernt, und so eine Reihe von Maschen bildet, der freilich aber auch oft um so weniger hervortritt, als er dicht an den Mittelnerv selbst sich anlegt.

Die Analogie dieser merkwürdigen Niederblätter mit denen von Hemitelia capensis Br. und Alsophila Boivini Mett. von Madagaskar (1. Hildebrand) scheint mir eine nähere als mit den Asplenien, die Karsten anführt. Bei diesen ist der morphologische Unterschied zwischen den Niederblättern und den Hochblättern lange nicht so stark, wie bei Acrostichum und 
Hemitelia. Eine ähnliche besondere Art von dimorphen Niederblättern bei Cyatheaceen bietet übrigens auch Alsophila ramispina Hook von Borneo l. Hose, bei welcher am Grunde der Wedel ein stark fiedergetheiltes, kurzes, Stipula-artiges Gebilde vorhanden ist, dessen Zipfel sich dornig verhärten.

III. Ueber Dimorphismus und Einsenkiung der Sori bei Polypodium. Karsten bemerkt S. 148, die alte Meinung, es handle sich bei den Polypodiumarten mit dimorphen Wedeln um eine Differenz fertiler und steriler Blattformen, sei dahin zu berichtigen, dass es sich bei diesem Dimorphismus nur um den Unterschied von Nischenblättern zum Zweck des Humussammels und von Vegetationsblättern handle. Hiegegen ist doch zu erinnern, dass es Polypodien giebt, bei denen ein starker Dimorphismus lediglich mit Bezug auf sterile und fertile Vegetationsblätter besteht. Im Malay. Archipel gehört dahin z. b. P. lomarioides Kze, P. caudiforme Bl. (Gymnogramme caudiformis Hook), P. incurvatum Bl., und zum Theil selbst gewisse Formen des gemeinen P. Phymatodes L. (siehe Dr. H. Christ filices Sarasinianae in Verh. der Naturforsch. Ges. Basel. XI. 1. Seite 72). Es ist sehr denkbar, dass die Fruchtwedel dieser Arten deshalb so viel schmäler sind als die sterilen, damit die Sori einer geringeren Kraftwirkung des zusammen strömenden, auf grossen Flächen doppelt schwer einwirkenden Regenwassers ausgesezt sind.

Bei mehrern Polypodien der Malay. Region kommt es vor, dass auf demselben Wedel der untere sterile Theil breite Abschnitte hat, während der obere Theil, so weit er fertil ist, ganz schmal und auf die Nerven nebst einem Flügel reducirt ist, gerade breit genug, um eine Sorus-Reihe zu tragen. Dies ist ein Dimorphismus innerhalb desselben Wedels. Dahin gehört Polypodium (Drynaria) splendens Hook (Dryostachyum pilosum J. Sm.) von Luzon und Celebes (1. F. und P. Sarasin). Die untere Hälfte des Wedels trägt breite Segmente, aber in der Mitte zieht sich der Wedel plötzlich so zusammen, dass er einer fertilen Lomoria mit sehr entfernten und langen Segmenten ähnlich ist. Dieselbe Gestaltung findet sich bei dem nahe verwandten Polyp. (Drynaria) Mayenianum (sub Aglao- 
morpha Schott) der Philippinen und endlich bei der, mit diesen Polypodien ebenfalls ganz nahe verwandten, aber aus künstlich theoretischen Gründen zu den Acrostichum geworfenen Photinopteris rigida J. Sm. und drynarioides Hooker. Dass bei den so überaus zahlreichen, wirklichen Acrostichum dieser Zone der Dimorphismus in Betreff der Ausdehnung der sterilen, und der Reduction der fertilen Lamina die Regel ist, braucht keiner Erwähnung: es kommt hier Dimorphismus mit getrennten Wedeln und auch solcher auf demselben Wedel vor, letzteres z. B. bei dem sehr verbreiteten und gemeinen Acrostichum (Hymenolepis) spicatum L. wo die breit bandförmige Lamina sich plötzlich in eine lineale fertile Spitze zusammen zieht: ohne Zweifel zum Schutz der Sporangienmasse gegen breite Regenströme.

Ich nehme an, dass auch gerade deshalb viele Polypodien aus der regenreichsten, mittlern Gebirgsregion des Malay. Archipels so tief eingesenkte Sori haben. Ein gutes Beispiel bietet Polyp. incurvatum Blume. Nicht nur sind die fertilen Wedel gegenüber den sehr breiten sterilen besonders schmal, fast auf schmal geflügelte Rippen reducirt, sondern die Sori sind so tief in die Blattunterseite eingesenkt, dass sie auf der Oberseite sackförmig heraustretende, nach unten verjüngte Papillen von 3-4 Millim. Tiefe bilden, auf deren Grunde sie ruhen. Auf diese Weise sind die bekanntlich sehr schwach, mit ihrem dünnsten Ende befestigten Sporangien völlig geschüzt gegen die Gewalt des strömenden Wassers, bis sie reifen und dann die Sporen durch die Elastizität der Sporangienringe über den Rand des schützenden Sacks hinausgeschleudert werden. Besonders merkwürdig war mir die von F. und P. Sarasin in der Bergregion von Celebes (Klabat-Gipfel) gesammelte, von Blume P. celebicum genannte Pflanze. Sie ist vom gemeinen $P$. pectinatum L. des warmen Amerika fast nur durch eingesenkte Sori zu unterscheiden, die ausserdem noch durch eine randliche Wimperbehaarung geschüzt sind, welche die Regentropfen zertheilt und abhält.

Ein weiteres Beispiel ist das im Archipel wie auch in Hinter- 
indien gemeine Polyp. subauriculatum Blume, das nicht dinorph ist, aber dessen Sori papillös eingesenkt sind. Die zwei und mehr Meter langen Wedel dieses sehr ornamentalen Farns hängen häufig senkrecht abwärts. Es ist klar, dass das strömende Wasser durch die Nische, in welcher die Sori geborgen sind, abgehalten wird.

Bei allen diesen Far'nen mit papillös eingebetteten Sori fehlt, gemäss ihrem Genuscharakter Polypodium, der Schleier, der andern Filices (Nephrodium, Asplenium etc.) zum Schutz dient. Bei einigen, und zwar breitblättrigen Aspidium kommt übrigens der Schutz durch eingesenkte Sori als Ausnahme auch vor: ich sah cultivirtes Aspidium trifoliatum dieser Art und besitze ein A. macrophyllum aus Centr. Amerika (Honduras C. Donnell-Smith) das diese Erscheinung in ausgesprochenem Grade zeigt, während normal diese Species flach aufsitzende Sori haben. Dass übrigens auch die Oberseite des Wedels durch die hervortretenden Papillen geschüzt ist, braucht keiner Erwähnung.

Mehrere Filices der Malay. Region zeigen den Schutz der Sori durch ein Indusium im höchsten Grade. Die im Auf bau Nephrodium ähnliche Diacalpe hat ein Indusium, das den Sorus völlig als Hohlkugel umschliesst, und die ähnliche Sphaeropteris zeigt dieselbe Erscheinung, aber so, dass die, den Sorus umschliessende Hohlkugel gestielt ist, wodurch natürlich der Sorus noch mehr dem Einfluss des auf der Blattfläche fortrollenden Wassers entrückt ist.

Bei der seltenen, den Gleichenien verwandten Mattonia von Sumatra und Borneo bedeckt ein gestielter, tief über den Rand des Sorus herabgreifender Schirm von sehr fester Textur denselben gegen Einwirkungen von oben sowohl als von del Seite. In andern genera sorgt die feine Zertheilung des Wedels genügend für Zertheilung der Wassermasse.

Ich schreibe dieser Zertheilung den Umstand zu, dass in den Genera Alsophila und Cyathea so höchst ähnliche Formen bald mit, bald ohne Indusium auskommen. Die Zertheilung des Laubes genügt zum Schutze, und die Bedeckung der Sori wird indifferent. Bemerkenswerth ist in dieser Richtung, dass 
eine Form: Alsophila tristis, Blume von den Bergen Java's (Gedeh und Tjibodas L. Lefèvre c. Treub) mit Diacalpe so sehr übereinstimmt, dass eine specifische Trennung kaum möglich ist, ausser dass sie indusiumlose Sori hat! Nahezu dieselbe Pflanze tritt also bald mit geschüztem und bald mit ungeschürtem Sorus auf, wie ich glaube weil die feine und also den Regen durchlassende Zertheilung des Wedels die Sori bereits genügend vor all zu starker Schwemmung durch Regen schüzt.

Die gitterförmige Zertheilung der doppelt- und dreifach gefiederten Wedel genügt auch bei sehr zarter Textur (und sowohl Diacalpe als Alsophila tristis sind von dünnem krautigen Gewebe) um die grosse Wedelfläche zu entlasten, weil sich keine dicke Regenschicht und keine starken Regenbäche darauf bilden können, sondern weil zwischen jeder Fieder und jedem Fiedersegment ein leerer Raum ist, durch welchen das Wasser herabfällt und so die Kraftcontinuität verliert.

Das Maximum des Schuzes ihrer Sori geniessen freilich Formen, wie z. B. Alsophila crinita Hook. Java's, Ceylons und Südindiens, die bei sehr straffem, lederigem Laub, sowie bei einer sehr weit gehenden Zertheilung noch mit steif abstehenden Spreuhaaren vollständig bekleidet ist. Hier kommt zu der elastischen, das Gewicht des Wassers durch die Federkraft der Spindel und des Gewebes zurückwerfenden Natur der Pflanze, und zu den geschilderten Vortheilen des Gitterwerks noch die Zerstäubung, welche die auffallenden Tropfen durch die sich ihnen entgegenstellende Behaarung erfahren.

Sehr interessant sind auch Formen, wo die Laubtheile zwar kahl sind, wo aber die Spindel und selbst die Rippen (Hauptnerven) der F'iedern mit dichten Spreublättchen (Cyathea Tussacii Desv. der Antillen) oder Wollhaaren (Cyathea glauca Bory von Bourbon 1. Cordemoy) geschüzt sind und zwar nicht nur in der Jugend, sondern bis ins Alter. Es handelt sich auch hier ohne Zweifel um die Hinderung allzu starker Zuströmung des Wassers. 
